\title{
Article
}

\section{Characterization of Folding Intermediates of a Domain-Swapped Protein by Solid-State NMR Spectroscopy}

Manuel Etzkorn, Anja Bckmann, Franois Penin, Dietmar Riedel, and Marc Baldus

J. Am. Chem. Soc., 2007, 129 (1), 169-175• DOI: 10.1021/ja066469x • Publication Date (Web): 15 December 2006

Downloaded from http://pubs.acs.org on March 24, 2009
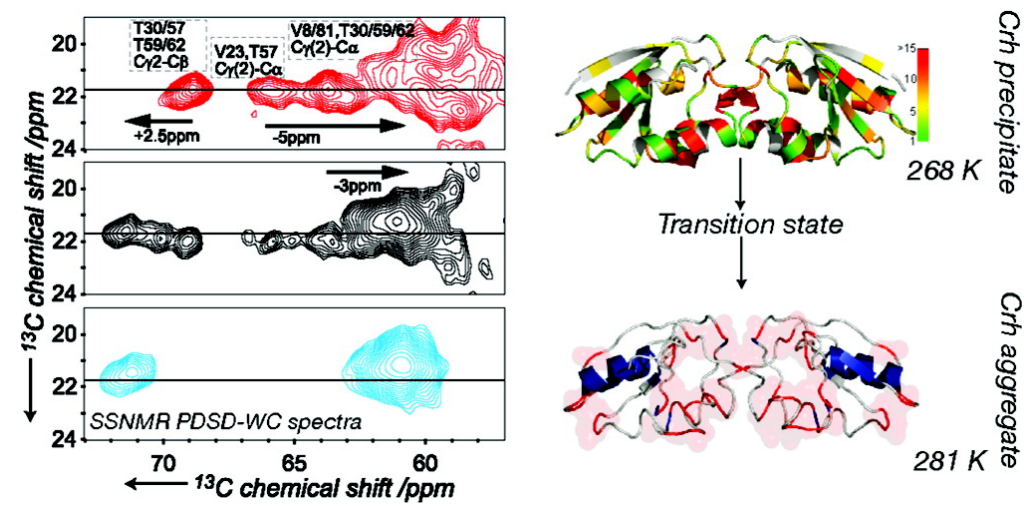

\section{More About This Article}

Additional resources and features associated with this article are available within the HTML version:

- $\quad$ Supporting Information

- $\quad$ Links to the 6 articles that cite this article, as of the time of this article download

- $\quad$ Access to high resolution figures

- $\quad$ Links to articles and content related to this article

- $\quad$ Copyright permission to reproduce figures and/or text from this article

\section{View the Full Text HTML}

\section{ACS Publications}




\title{
Characterization of Folding Intermediates of a Domain-Swapped Protein by Solid-State NMR Spectroscopy
}

\author{
Manuel Etzkorn, ${ }^{\dagger}$ Anja Böckmann, ${ }^{*}$, François Penin, ${ }^{\S}$ Dietmar Riedel, ${ }^{\ddagger}$ and \\ Marc Baldus*, \\ Contribution from the Departments of NMR-Based Structural Biology and Electron Microscopy, \\ Max-Planck-Institute for Biophysical Chemistry, Am Fassberg 11, 37077 Göttingen, Germany, \\ and Institut de Biologie et Chimie des Protéines, UMR 5086 CNRS-Université Lyon 1, IFR128 \\ BioSciences Lyon-Gerland, 7, passage du Vercors, 69367 Lyon, France
}

Received September 7, 2006; E-mail: a.bockmann@ibcp.fr; maba@mpibpc.mpg.de

\begin{abstract}
We have employed two-dimensional solid-state NMR to study structure and dynamics of insoluble folding states of the domain-swapped protein Crh. Starting from the protein precipitated at its $\mathrm{pl}$, conformational changes due to a modest temperature increase were investigated at the level of individual residues and in real-time. As compared to the crystalline state, Crh pl-precipitates exhibited a higher degree of molecular mobility for several regions of the protein. A rigidly intact center was observed including a subset of residues of the hydrophobic core. Raising the temperature by $13 \mathrm{~K}$ to $282 \mathrm{~K}$ created a partially unfolded intermediate state that was converted into $\beta$-sheet-rich aggregates that are mostly of spherical character according to electron microscopy. Residue-by-residue analysis indicated that two out of three $\alpha$-helices in aggregated Crh underwent major structural rearrangements while the third helix was preserved. Residues in the hinge region exhibited major chemical-shift changes, indicating that the domain swap was not conserved in the aggregated form. Our study provides direct evidence that protein aggregates of a domain-swapped protein retain a significant fraction of native secondary structure and demonstrates that solid-state NMR can be used to directly monitor slow molecular folding events.
\end{abstract}

\section{Introduction}

Understanding the ways in which proteins or protein fragments self-assemble into ordered, insoluble aggregates associated with amyloid diseases is of paramount biophysical and medical interest. ${ }^{1}$ Delineating the mechanisms of protein folding and aggregation and their possible control by pharmacological intervention requires the identification of key species involved in folding and aggregation and the precise definition of their roles in each pathway. The final, and possibly also earlier, intermediate protein states are often insoluble. This complicates the application of well-established techniques to study biomolecules at atomic resolution, that is, liquid-state NMR and X-ray crystallography. Solid-state NMR (ssNMR) has recently made significant progress in studying nonnative protein states ${ }^{2-6}$ and protein folding ${ }^{7,8}$ at the molecular level. Indeed, ssNMR

$\dagger$ Department of NMR-Based Structural Biology, Max-Planck-Institute for Biophysical Chemistry.

‡ Department of Electron Microscopy, Max-Planck-Institute for Biophysical Chemistry.

$\S$ Institut de Biologie et Chimie des Protéines.

(1) Dobson, C. M. Nature 2003, 426, 884-890.

(2) Tycko, R. Curr. Opin. Struct. Biol. 2004, 14, 96-103.

(3) Siemer, A. B.; Ritter, C.; Ernst, M.; Riek, R.; Meier, B. H. Angew. Chem., Int. Ed. 2005, 44, 2441-2444.

(4) Ritter, C.; Maddelein, M.-L.; Siemer, A. B.; Luhrs, T.; Ernst, M.; Meier, B. H.; Saupe, S. J.; Riek, R. Nature 2005, 435, 844-848.

(5) Heise, H.; Hoyer, W.; Becker, S.; Andronesi, O. C.; Riedel, D.; Baldus, M. Proc. Natl. Acad. Sci. U.S.A. 2005, 102, 15871-15876.

(6) Petkova, A. T.; Ishii, Y.; Balbach, J. J.; Antzutkin, O. N.; Leapman, R. D.; Delaglio, F.; Tycko, R. Proc. Natl. Acad. Sci. U.S.A. 2002, 99, $16742-$ 16747.

10.1021/ja066469x CCC: $\$ 37.00$ @ 2007 American Chemical Society spectroscopy can be performed in fully fibrillized ${ }^{2-6}$ or in freezetrapped $^{7-9}$ biomolecules; we show here that ssNMR also offers a spectroscopic means to follow protein folding events in realtime. While NMR has already successfully been applied for protein folding in solution, ${ }^{10}$ we demonstrate how highresolution ssNMR methods can be used to study conformational transitions between insoluble native and non-native states of a protein.

Our study focuses on the Crh protein from B. Subtilis. While its specific function remains elusive, ${ }^{11} \mathrm{Crh}$ has been shown to exist in remarkably different conformations. In solution, $\mathrm{Crh}$ forms a mixture of monomers and dimers in a slowly (i.e., time scale of hours) exchanging equilibrium. ${ }^{12}$ The monomer structure was resolved by liquid-state NMR. ${ }^{13}$ X-ray crystallography revealed a $3 \mathrm{D}$ domain-swapped dimer structure formed by $\beta 1$ strand swapping of two monomers. ${ }^{14}$ Domain swapping has been

(7) Havlin, R. H.; Tycko, R. Proc. Natl. Acad. Sci. U.S.A. 2005, 102, 32843289.

(8) Chimon, S.; Ishii, Y. J. Am. Chem. Soc. 2005, 127, 13472-13473.

(9) Heise, H.; Luca, S.; de Groot, B. L.; Grubmueller, H.; Baldus, M. Biophys. J. 2005, 89, 2113-2120.

(10) Balbach, J.; Forge, V.; Lau, W. S.; van Nuland, N. A. J.; Brew, K.; Dobson, C. M. Science 1996, 274, 1161-1163.

(11) Deutscher, J.; Galinier, A.; Martin-Verstraete, I. In Bacillus subtilis and its closest relatives: From Genes to Cells; Sonenschein, A. L., Hoch, J. A., Losick, R., Eds.; ASM Press: Washington, 2001; pp 129-150.

(12) Penin, F.; Favier, A.; Montserret, R.; Brutscher, B.; Deutscher, J.; Marion, D.; Galinier, A. J. Mol. Microbiol. Biotechnol. 2001, 3, 429-432.

(13) Favier, A.; Brutscher, B.; Blackledge, M.; Galinier, A.; Deutscher, J.; Penin, F.; Marion, D. J. Mol. Biol. 2002, 317, 131-144.

(14) Juy, M.; Penin, F.; Favier, A.; Galinier, A.; Montserret, R.; Haser, R.; Deutscher, J.; Böckmann, A. J. Mol. Biol. 2003, 332, 767-776. 


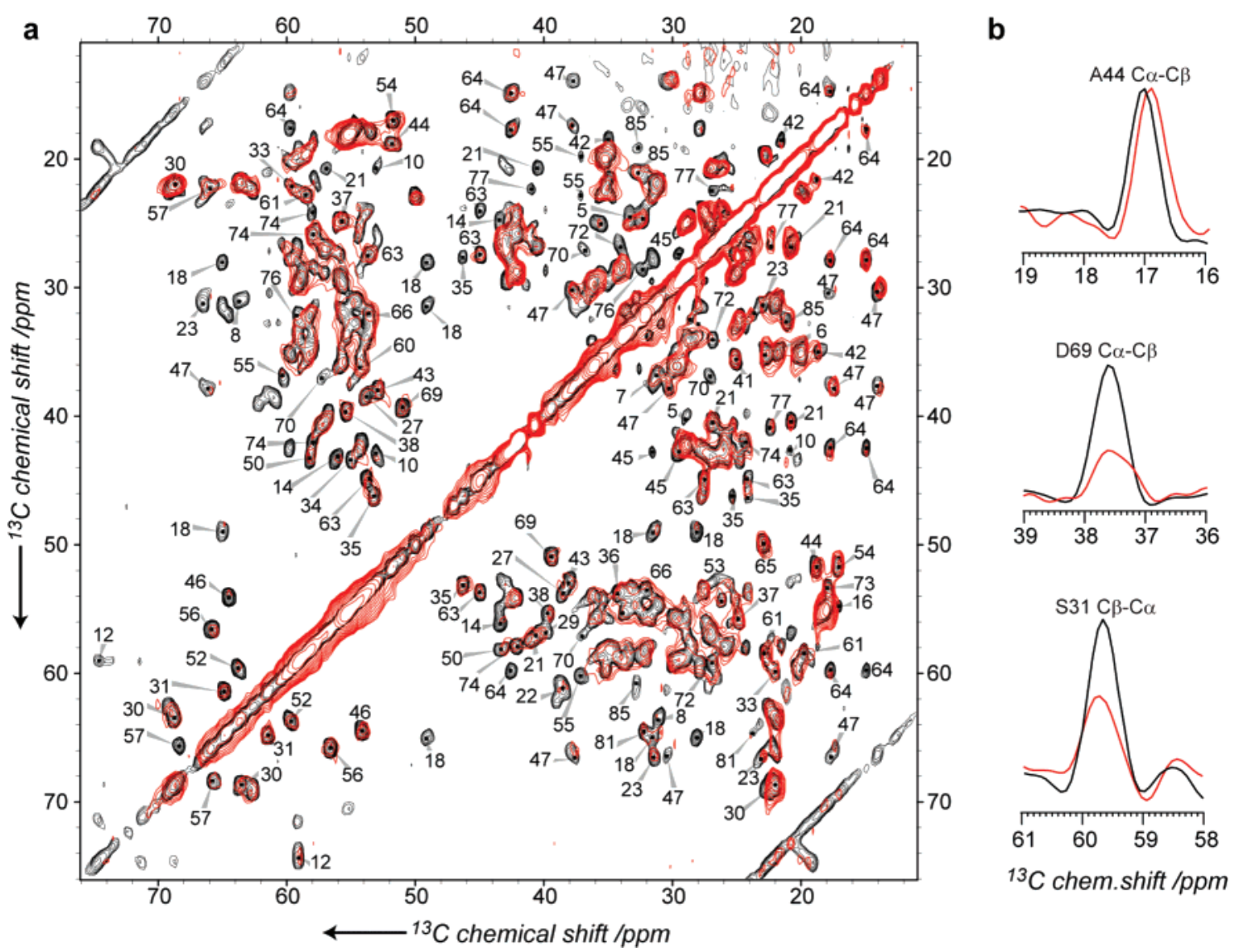

Figure 1. Comparison of $2 \mathrm{D}{ }^{13} \mathrm{C}-{ }^{13} \mathrm{C}$ PDSD spectra (aliphatic region) obtained on microcrystalline (black) and pI-precipitated (red) Crh (a). Extracts shown in (b) exemplify variations in $\mathrm{C} \alpha-\mathrm{C} \beta$ cross-peak amplitudes for the indicated residues. Both spectra were taken under identical experimental conditions (see Supporting Information). Assignments are according to ref 28.

forwarded as a mechanism for protein oligomerization, ${ }^{15-19}$ possibly involving the native protein state, ${ }^{19}$ and has been considered as a possible means for functional regulation. ${ }^{18,20,21}$ It may also be closely associated with misfolding and aggregation, as has been suggested in the context of human prion protein $^{16}$ and amyloidogenic human cystatin C. ${ }^{22}$ A variety of biophysical methods, computational studies ${ }^{23-25}$ and solutionstate NMR, ${ }^{26}$ as well as mutagenesis ${ }^{27}$ have been employed to elucidate structural details associated with folding of a monomeric protein into a domain-swapped form. However, direct structural information about insoluble intermediate and fully aggregated folding states and their respective lifetimes has been lacking.

(15) Rousseau, F.; Schymkowitz, J. W. H.; Itzhaki, L. S. Structure 2003, 11, $243-251$

(16) Knaus, K. J.; Morillas, M.; Swietnicki, W.; Malone, M.; Surewicz, W. K.; Yee, V. C. Nat. Struct. Biol. 2001, 8, 770-774.

(17) Louis, J. M.; Byeon, I. J.; Baxa, U.; Gronenborn, A. M. J. Mol. Biol. 2005, $348,687-698$.

(18) Bennett, M. J.; Schlunegger, M. P.; Eisenberg, D. Protein Sci. 1995, 4, $2455-2468$.

(19) Sambashivan, S.; Liu, Y.; Sawaya, M. R.; Gingery, M.; Eisenberg, D. Nature $\mathbf{2 0 0 5}, 437,266-269$

(20) Bennett, M. J.; Choe, S.; Eisenberg, D. Protein Sci. 1994, 3, 1444-1463.

(21) Newcomer, M. E. Curr. Opin. Struct. Biol. 2002, 12, 48-53.

(22) Janowski, R.; Kozak, M.; Jankowska, E.; Grzonka, Z.; Grubb, A.; Abrahamson, M.; Jaskolski, M. Nat. Struct. Biol. 2001, 8, 316-320.

(23) Yang, S.; Cho, S. S.; Levy, Y.; Cheung, M. S.; Levine, H.; Wolynes, P. G.; Onuchic, J. N. Proc. Natl. Acad. Sci. U.S.A. 2004, 101, 13786-13791.

(24) Esposito, L.; Daggett, V. Biochemistry 2005, 44, 3358-3368.

(25) Chahine, J.; Cheung, M. S. Biophys. J. 2005, 89, 2693-2700.

(26) Byeon, I. J.; Louis, J. M.; Gronenborn, A. M. J. Mol. Biol. 2004, 340, $615-25$.

(27) Rousseau, F.; Schymkowitz, J. W.; Wilkinson, H. R.; Itzhaki, L. S. Proc. Natl. Acad. Sci. U.S.A. 2001, 98, 5596-5601.
Previously, we have shown by ssNMR that Crh domainswapped dimers are already present in the microcrystalline state. ${ }^{28,29}$ Here, we describe the spectroscopic investigation of conformational states obtained by precipitation of $\mathrm{Crh}$ at a $\mathrm{pH}$ of 6.5 corresponding to its isoelectric point, at which its solubility is minimal. We refer in the following to this preparation as pI-precipitated Crh. ssNMR data obtained on the precipitate are consistent with an overall domain-swapped structure in which major protein segments are destabilized and only part of the hydrophobic core is rigidly intact. We show that a folding event can be induced by a modest temperature increase, which is monitored by $2 \mathrm{D}$ ssNMR in real-time. Analysis of the corresponding 2D ssNMR correlation spectra acquired before, during, and after the folding event is consistent with a folding pathway starting at a dynamically destabilized domain-swapped dimer. Upon temperature increase, a partially unfolded intermediate state was created that directly led to protein aggregates not only rich in $\beta$-sheet secondary structure but also preserving significant $\alpha$-helical segments. Our study (i) reveals that molecular dynamics may play an important role in protein aggregation, (ii) provides direct evidence for a partially unfolded intermediate state in a domain-swapped dimer, and (iii) demonstrates the possibility to structurally analyze aggregated protein states in a site-resolved manner.

(28) Böckmann, A.; Lange, A.; Galinier, A.; Luca, S.; Giraud, N.; Juy, M.; Heise, H.; Montserret, R.; Penin, F.; Baldus, M. J. Biomol. NMR 2003, 27, 323-339.

(29) Etzkorn, M.; Böckmann, A.; Lange, A.; Baldus, M. J. Am. Chem. Soc. 2004, 126, 14746-14751

170 J. AM. CHEM. SOC. - VOL. 129, NO. 1, 2007 


\section{Materials and Methods}

(a) Sample Preparation. Microcrystalline Crh was prepared as described previously. ${ }^{28}$ The pI-precipitated Crh sample was obtained by changing the $\mathrm{pH}$ of a protein solution $(20 \mathrm{mg} / \mathrm{mL})$ to the isoelectric $\mathrm{pH}(\mathrm{pI}=6.5)$ by addition of $\mathrm{HCl} 1 \mathrm{M}$. Precipitation occurred instantaneously, and the sample was left in the freezer for 5 days to complete the process. The precipitate was transferred into a $4 \mathrm{~mm}$ CRAMPS rotor, and the rotor cap was sealed.

(b) Electron Microscopy (EM). Crh aggregates were diluted 1:50 with $\mathrm{NH}_{4} \mathrm{CO}_{3}$ buffer and prepared on a glow discharged carbon foil. After being stained with $1 \%$ uranyl acetate, the samples were evaluated with a CM 200 transmission electron microscope (FEI, Eindhoven, and The Netherlands). Pictures were taken with a TemCam F415A camera (TVIPS, Gauting, Germany) at 20000 -fold magnification.

(c) Solid-State NMR Spectroscopy and Data Analysis. All NMR experiments were conduced using $4 \mathrm{~mm}$ triple-resonance probeheads at static magnetic fields of 18.8 and $14.1 \mathrm{~T}$ corresponding to 800 and $600 \mathrm{MHz}{ }^{1} \mathrm{H}$ resonance frequency (Bruker Biospin, Karlsruhe/ Germany). Broadband $\left({ }^{1} \mathrm{H},{ }^{13} \mathrm{C}\right)$ and chemical-shift selective $\left({ }^{15} \mathrm{~N},{ }^{13} \mathrm{C}\right)$ Hartmann-Hahn cross polarization (SPECIFIC-CP ${ }^{30}$ ) steps were applied using SPINAL64 ${ }^{31}$ proton decoupling with radio frequency fields of $75-90 \mathrm{kHz}$ during evolution $\left(t_{1}\right)$, detection $\left(t_{2}\right)$, and $\mathrm{N}-\mathrm{C}$ transfer. For intra-residue $\mathrm{C}-\mathrm{C}$ transfer, PDSD (proton-driven spin diffusion) with a mixing time of $20 \mathrm{~ms}$ was used. Sequential $\left(\mathrm{C} x_{i}-\mathrm{C} y_{i \pm 1}\right)$ magnetization transfer was achieved using spin diffusion under weak coupling conditions ${ }^{32}$ with mixing times between 80 and $150 \mathrm{~ms}$. All spectra were processed using QSINE window functions in F1 and F2 and analyzed with Sparky version 3.110 (T. D. Goddard, D. G. Kneller, University of California). Protein structures were visualized using Pymol (DeLano Scientific, San Carlos, CA).

\section{Results}

(a) Comparison of Microcrystalline/pI-Precipitated Crh. In Figure 1, we compare homonuclear ${ }^{13} \mathrm{C}$ PDSD spectra of the Crh pI-precipitate at $269 \mathrm{~K}$ (red) to results using a microcrystalline preparation ${ }^{28}$ (black). The spectra clearly show that the general chemical-shift pattern is conserved and ${ }^{13} \mathrm{C}$ line widths are comparable for most signals. Similar observations were made for $2 \mathrm{D}{ }^{13} \mathrm{C}-{ }^{15} \mathrm{~N}$ heteronuclear correlation spectra (see Supporting Information), albeit with a larger ${ }^{15} \mathrm{~N}$ line width. The chemical-shift differences found between the precipitated and the microcrystalline forms of $\mathrm{Crh}$ are small in general: only some residues show changes of about $0.5 \mathrm{ppm}$, and a few up to around $1 \mathrm{ppm}$ (see Supporting Information). Even without sequential assignments for the precipitate, chemical-shift changes can be traced for most residues, and no new cross signals are observed. The domain swap involving $\beta$-strand 1 can still be identified by the characteristic chemical shift of Thr12 located in the hinge region (Figure 1a). The high similarity of chemical shifts observed between the microcrystalline $\mathrm{Crh}$ and the precipitated form indicates that the global fold of $\mathrm{Crh}$ is conserved in the precipitate.

The most remarkable difference between the spectra of microcrystalline and precipitated $\mathrm{Crh}$ lies in the signal intensities. As exemplified in Figure $1 \mathrm{~b}$, the ssNMR line width is comparable, even when signal intensities are largely attenuated. Thus, static disorder (leading to inhomogenous broadening) is not the source of the observed signal loss, and variations in

(30) Baldus, M.; Petkova, A. T.; Herzfeld, J.; Griffin, R. G. Mol. Phys. 1998, 95, 1197-1207.

(31) Fung, B. M.; Khitrin, A. K.; Ermolaev, K. J. Magn. Reson. 2000, 142, 97-101

(32) Seidel, K.; Lange, A.; Becker, S.; Hughes, C. E.; Heise, H.; Baldus, M. Phys. Chem. Chem. Phys. 2004, 6, 5090-5093. a
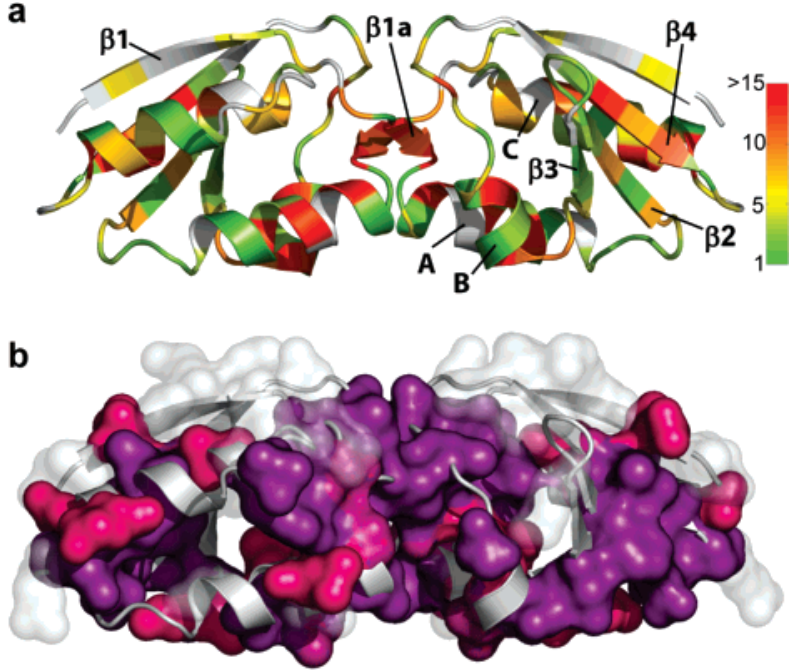

C

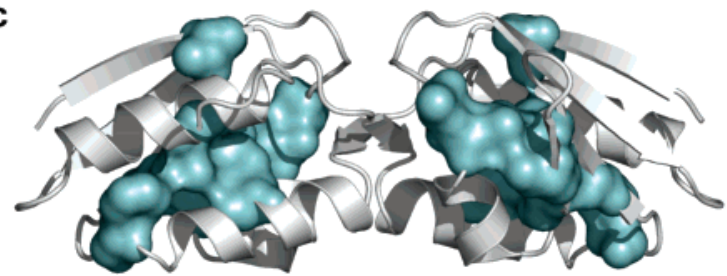

Figure 2. (a) X-ray structure of the Crh domain-swapped dimer (PDB code $1 \mathrm{mu} 4^{14}$ ) with color gradients corresponding to the observed cross signal attenuation in spectra of $\mathrm{pI}$-precipitated $\mathrm{Crh}$ as compared to the spectrum of the microcrystalline sample, ranging from strong $\left(I_{\mathrm{m}} / I_{\mathrm{p}}>15\right.$, red) to no $\left(I_{\mathrm{m}} / I_{\mathrm{p}}=1\right.$, green) attenuation. Not observed: white. The largest attenuation detected for a resonance belonging to a specific residue is reported. Helices $\mathrm{A}-\mathrm{C}$ and $\beta$-strands $\beta 1-\beta 4$ are indicated. In (b), residues that exhibit attenuated cross signals $\left(I_{\mathrm{m}} / I_{\mathrm{p}}>5\right)$ for resonances involving $\mathrm{C}^{\prime}-\mathrm{C} \alpha$ and $\mathrm{C} \alpha-\mathrm{C} \beta$ cross signals are shown in (surface-mode) purple, and cross signals involving side-chain carbons are in pink. Residues for which no information could be obtained due to signal overlap are colored in transparent gray. In (c), the static residues of the hydrophobic core are shown in cyan. All other protein segments are given in white.

signal intensities can be attributed to variations in molecular mobility. For residues with resolved cross signals, we determined the relative intensity ratio $I_{\mathrm{m}} / I_{\mathrm{p}}$ of ssNMR signals obtained on microcrystalline and pI-precipitated Crh (see Figure 1 and Supporting Information Figure 7b). High ratios indicate a higher degree of local mobility in the pI-precipitated sample, which is a priori not constrained by a crystal lattice. These parameters are mapped on the ribbon diagram of the domain-swapped crystal structure of $\mathrm{Crh}$ in Figure $2 \mathrm{a}$ (PDB code $1 \mathrm{mu} 4^{14}$ ). The degree of signal attenuation ranges from green (rigid, $I_{\mathrm{m}} / I_{\mathrm{p}} \approx$ 1) to red (strongest dynamics, $I_{\mathrm{m}} / I_{\mathrm{p}}>15$ ). Residues not identified are given in white. In Figure $2 b$, assigned protein residues exhibiting sizable dynamics $\left(I_{\mathrm{m}} / I_{\mathrm{p}}>5\right.$ in a) on the backbone (purple) or side-chain (pink) level are shown in surface mode. The attenuation of hydrophilic side-chain signals from residues located at the surface comes without surprise. Interestingly, many of the backbone signals, and also signals from residues being part of the hydrophobic core of $\mathrm{Crh}$, are affected. Moreover, significant cross signal attenuation is observed around the active site (residues Lys45, Ser46, and Ile47) and for several residues in helix A (residues Ala16, Pro18, Leu21, Val23). The most affected secondary structure element is, however, the $\beta 1 \mathrm{a}-$ strand, which forms the hinge region in the domain-swapped dimer (residues Thr12-Leu14). Another region where dynamic variations concentrate is the end of $\beta$-strand 4 , and beginning 

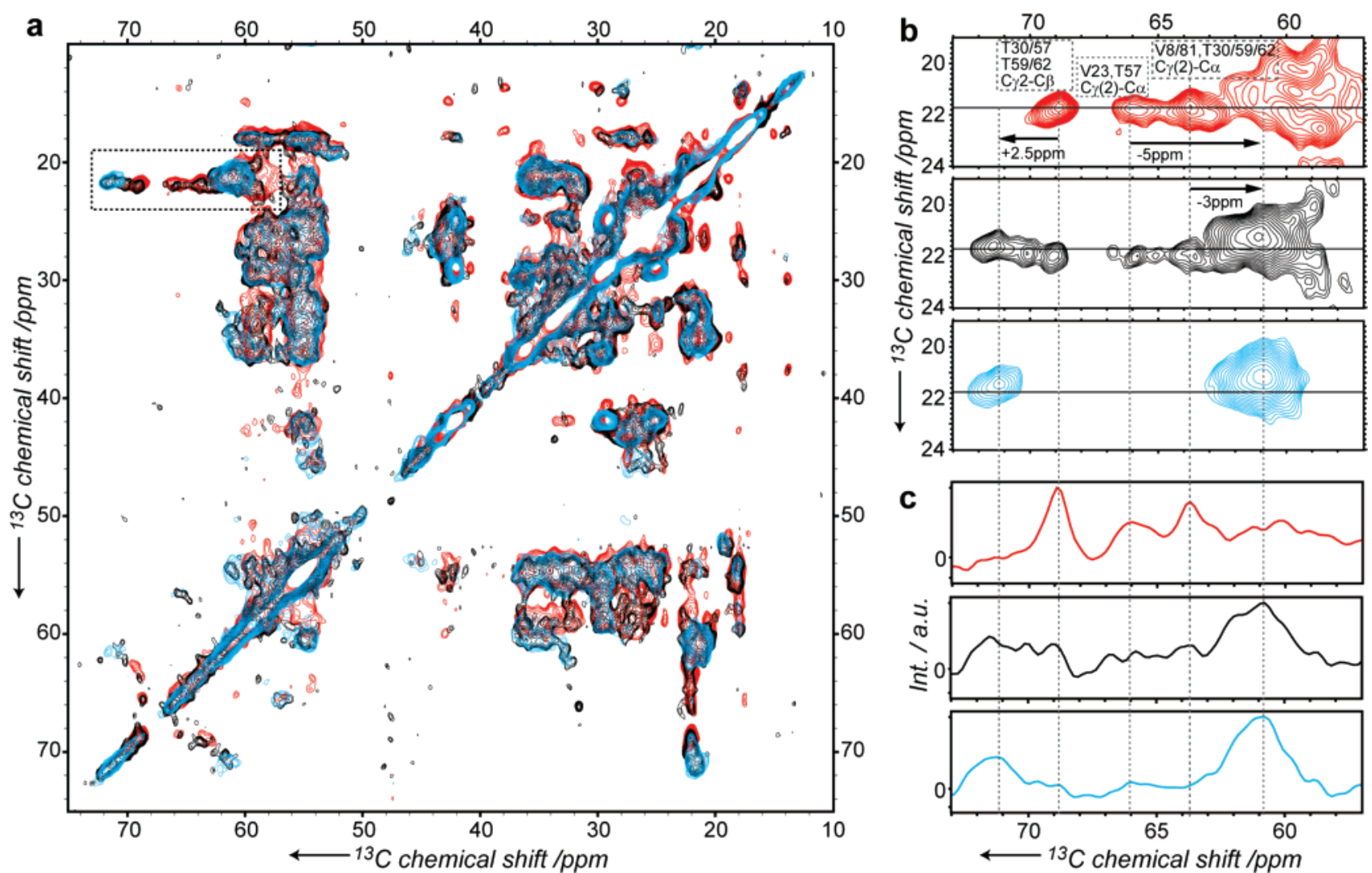

Figure 3. Time-resolved 2D ssNMR experiments. (a) Overlay of three spectra: red, pI-precipitated Crh $(T=269 \mathrm{~K})$; black, recorded immediately after a temperature increase to $T=282 \mathrm{~K}$; blue, 1 day after temperature raise $(T=282 \mathrm{~K})$. Spectra of the highlighted region are shown separately in (b). In (c), cross-peak intensities of the frequency indicated by horizontal lines (in (b)) are plotted. The spectra were recorded at $800 \mathrm{MHz}$, at a spinning frequency of $12.5 \mathrm{kHz}$. Magnetization transfer was achieved using spin diffusion under weak-coupling conditions ${ }^{32}$ with a mixing time of $150 \mathrm{~ms}$. A side-by-side view of the spectra is shown in Supporting Information Figure 8.

of helix C (residues Leu63-Leu74). Notably, in a mutant form of the homologous protein HPr (histidine-containing phosphocarrier protein), $\beta$-strand 4 and helix $\mathrm{C}$ are able to undergo important structural changes. ${ }^{33}$ Indeed, a destabilization in this part of the protein in the Hpr F29W mutant leads to a domainswapped form of HPr, involving the C-terminal part of the protein, including $\beta$-strand 4 and helix C. The Asp69 residue, equally conserved in $\mathrm{Crh}$, and located in the loop between strand 4 and helix C, plays a central role in the folding mechanism of Hpr. ${ }^{34}$ In Crh, the Asp69 side chain is involved in hydrogen bonds with the amide and hydroxyl protons of residues Thr30 and Ser31, as well as with Ser31 $\mathrm{H} \gamma$. Both Asp69 and Ser31 residues show attenuated cross-peak intensities, indicating a destabilization of this important contact.

In Figure $2 b$, the empty pocket in the center of each monomer reveals a dynamically stable subset of the hydrophobic core illustrated in cyan in Figure 2c. Among these residues, only two are found on the $\mathrm{N}$ - or $\mathrm{C}$-terminal part of the protein. On the other hand, residues forming part of the classical hydrophobic core and situated in the N-terminus, including Val8, Leu10, and Leu14, or in the C-terminal $\beta$-strand 4 and helix C, like Leu63, Ala73, Leu74, and Val81, are not part of this rigid core. Notably, these observations are in line with Eisenberg et al., ${ }^{35}$ who postulated that any protein can swap domains under appropriate conditions, as long as the terminal domain of the protein is unconstrained.

(33) Sridharan, S.; Razvi, A.; Scholtz, J. M.; Sacchettini, J. C. J. Mol. Biol. 2005, 346, 919-931.

(34) Schmittschmitt, J. P.; Scholtz, J. M. Biochemistry 2004, 43, 1360-1368.

(35) Liu, Y.; Eisenberg, D. Protein Sci. 2002, 11, 1285-1299. (b) Refolding of Crh pI-Precipitate Monitored by TimeResolved Solid-State NMR. To further analyze the conformational space adopted by a domain-swapped protein, we recorded 2D ssNMR spectra immediately after a temperature rise from 269 to $282 \mathrm{~K}$. Figure 3 shows spectra of the $\mathrm{U}\left[{ }^{13} \mathrm{C},{ }^{15} \mathrm{~N}\right] \mathrm{Crh}$ precipitate recorded before (red), after the temperature increase (black), and 1 day afterward (blue). Interestingly, chemicalshift differences can be detected in several regions of the ssNMR spectrum, which can be most easily followed for the $\mathrm{C} \alpha / \mathrm{C} \beta$ spectral region involving $\mathrm{Thr}$ and Ser residues. As visible from the extracted region in Figure $3 b$, degenerate Thr $\mathrm{C} \beta / \mathrm{C} \gamma 2$ cross signals around 69/22 ppm are shifting along the $\omega_{2}$ dimension to lower field, resulting in a significant chemical-shift difference of about $2.5 \mathrm{ppm}$ for the $\operatorname{Thr} \mathrm{C} \beta$ atoms. Even stronger variations are seen for $\mathrm{C} \alpha-\mathrm{C} \gamma$ correlations that exhibit resonance shifts of $5 \mathrm{ppm}$. The Thr cross-peaks around $70 \mathrm{ppm}$ present in the 2D spectrum recorded immediately after the temperature increase (black) clearly indicate that not only the initial and final folding states are present in the spectrum. The corresponding partially resolved spectral "footprints" strongly suggest the presence of at least one transition state that differs from the initial and final state and exhibits a lifetime significantly longer than the duration of one $t_{1}$ experiment, that is, $5.5 \mathrm{~min}$. Indeed, protein conformations and folding intermediates different from the initial state will only be detectable in real-time if their resonances are distinct from those of the initial state and if the rate constants for their formation and disappearance result in a significant population of a given species on the time scale of our ssNMR experiment. In this case, solid-state NMR signals 


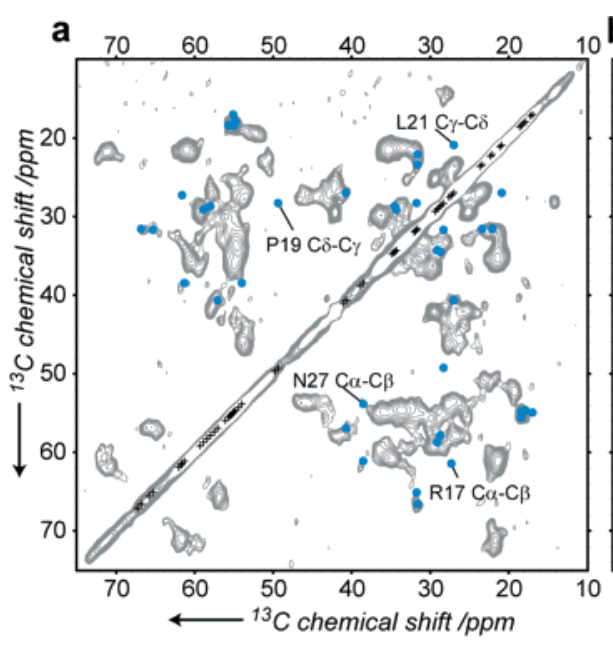

d

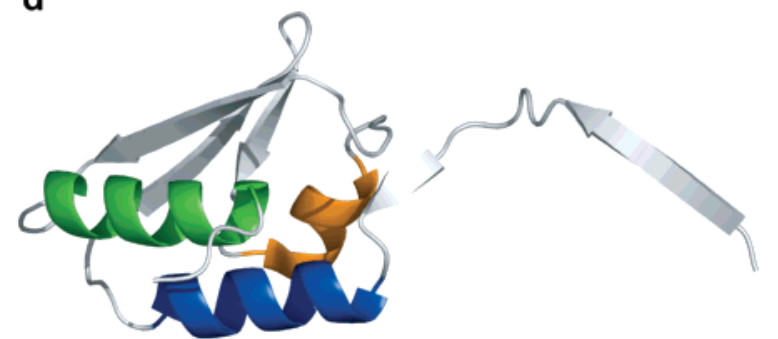

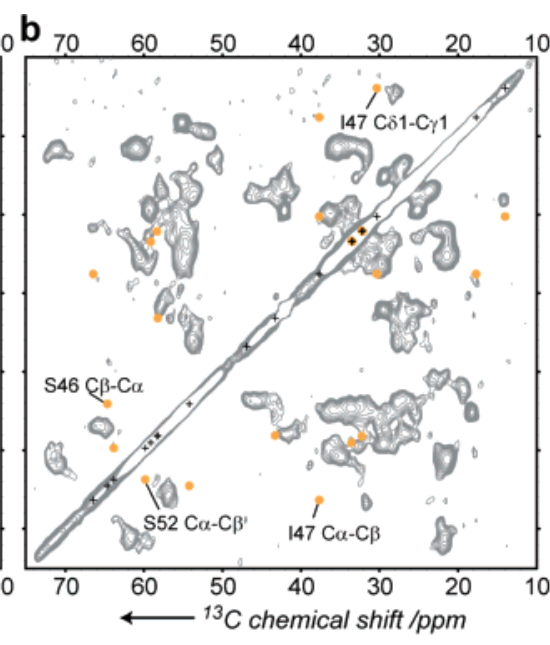

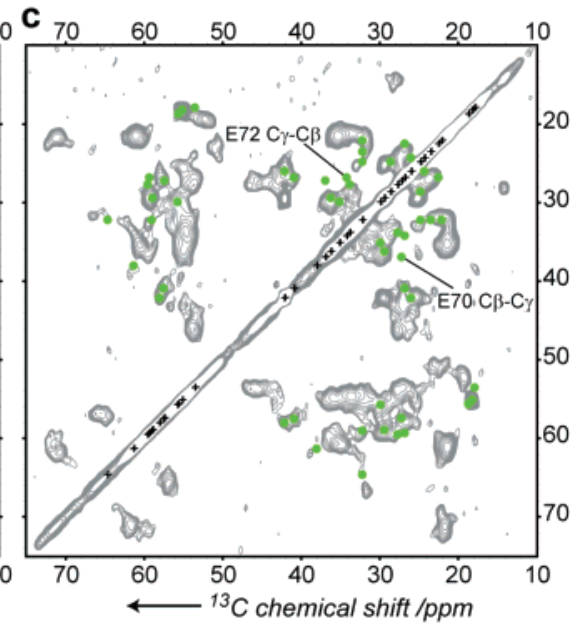

e

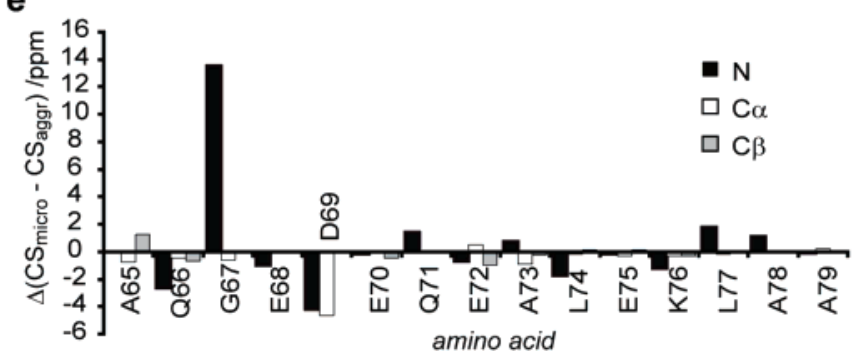

Figure 4. $2 \mathrm{D}{ }^{13} \mathrm{C}-{ }^{13} \mathrm{C}$ PDSD spectrum of aggregated $\mathrm{Crh}$. The spectrum was recorded at $600 \mathrm{MHz}{ }^{1} \mathrm{H}$ frequency, at a spinning speed of $10.5 \mathrm{kHz}$ and at $274 \mathrm{~K}$. PDSD with a mixing time of $20 \mathrm{~ms}$ was used for magnetization transfer. In (a), one-bond correlations for helix A assuming a microcrystalline dimer structure are shown in blue; in (b) and (c), the corresponding signal sets are given for helices B and C in yellow and green, respectively. (d) Location of the three helices A (blue), B (orange), and C (green) in one Crh monomer as seen in the crystal structure. ${ }^{14}$ (e) Chemical-shift changes $\Delta\left(\mathrm{CS}_{\text {micro }}-\mathrm{CS}_{\text {aggr }}\right)$ observed in NCACX and NCOCX spectra (relative to ref 28) for the sequential walk concerning residues 65-79. Note that helix C starts at residue E70.

from the different species in the frequency domain (F1) spectrum after double Fourier transformation will be convoluted by their kinetic profile in the folding reaction. These findings are corroborated by additional $\mathrm{C} \alpha / \mathrm{C} \beta$ cross-peak signals for Ser, Thr, and Pro, which exhibit chemical shifts typically associated with coil conformations in soluble proteins.

In the final state (third panel, Figure 3b), maximum intensity is found for the Thr $\mathrm{C} \beta$ residues around $71.5 \mathrm{ppm}$. The signals corresponding to the Val8 and $\mathrm{Val} 23 \mathrm{C} \alpha / \mathrm{C} \gamma$, and also to the overlapping Thr30/59/62 C $\alpha / \mathrm{C} \gamma 2$ and Thr57 $\mathrm{C} \alpha / \mathrm{C} \gamma 2$, respectively, are shifted upfield by about 3 and $5 \mathrm{ppm}$. Only very moderate intensity remains around 66 ppm, as shown in Figure $3 \mathrm{c}$, panel three. The observed chemical-shift changes remained the same in subsequent spectra recorded several weeks later (see, e.g., Figure 4 for a spectrum recorded 3 weeks after the transition). An upfield shift in $\mathrm{C} \alpha$ chemical shifts, combined with a downfield shift in $\mathrm{C} \beta$ chemical shifts, is indicative of an increase in $\beta$-strand conformation after the temperature increase. The corresponding $\mathrm{Crh}$ form was investigated by electron microscopy, which reveals mostly spherical aggregates (vide infra). Such aggregates have also been observed for precursor forms of amyloid-like aggregates. ${ }^{36}$

(c) Structural Analysis of the Aggregated Form. Although the ${ }^{13} \mathrm{C}$ line width seen for correlations in the aggregated state is comparable to spectra recorded before the temperatureinduced conformational transition, sequential resonance assignments are difficult to obtain for the final state. Instead, we

(36) Kayed, R.; Head, E.; Thompson, J.; McIntire, T.; Milton, S.; Cotman, C.; Glabe, C. Science 2003, 300, 486-489. compared the chemical shifts of the individual residues in microcrystalline Crh to data obtained on the aggregated form. Disappearing signals indicate either increased dynamics or conformational changes. Speaking in favor for the later, additional $\mathrm{C} \alpha-\mathrm{C} \beta$ cross signals with typical $\beta$-strand chemical shifts as well as a general increase in $\beta$-strand conformation was observed. Some $\alpha$-helical secondary structure is, however, still present, as exemplified by the occurrence of $\alpha$-helical chemical shifts, clearly observable for Ala residues (see below). Because the induced temperature increase is moderate, we assumed that these shifts reflect $\alpha$-helical protein segments already present before the transition. Correspondingly, the ssNMR analysis of the microcrystalline Crh sample could be used as a reference for determination of $\alpha$-helical segments in the aggregate. In particular, we examined which $\alpha$-helices are conserved in the aggregated form of the protein. Figure $4 a-c$ shows the PDSD spectrum of the aggregated form, as compared to resonance assignments obtained for the microcrystalline protein for helix A (Figure 4a, blue), helix B (Figure 4b, orange), and helix $\mathrm{C}$ (Figure 4c, green), respectively.

For $33 \%$ of residues in helix A (Ala16-Asn27) and $43 \%$ residues in helix B (Ser46-Ser52), at least one of the corresponding signals is no longer observed in $\mathrm{Crh}$ aggregates. On the contrary, all signals could be identified for helix $\mathrm{C}$ (residues Glu70-Gln82), with the exception of only two side-chain resonances. The high conservation of cross signals from helix $\mathrm{C}$ is also confirmed in NCACX and NCOCACX spectra (see Supporting Information Figure 9). Figure 4e shows the chemicalshift changes between aggregated and microcrystalline Crh 


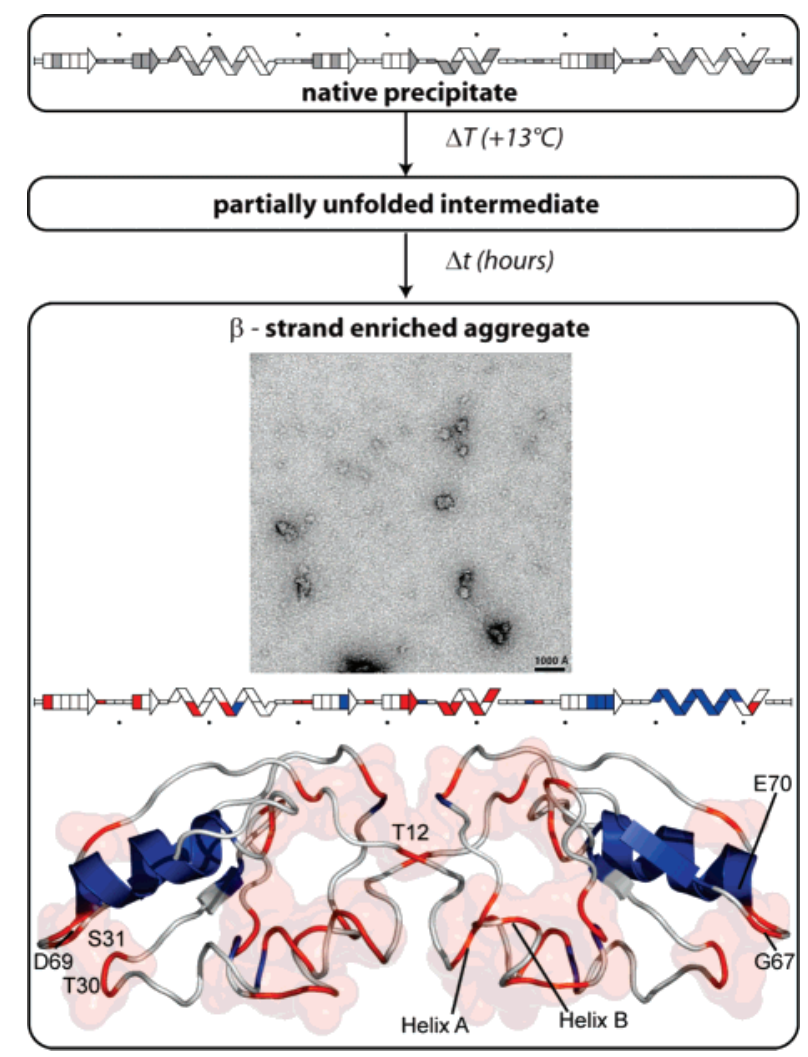

Figure 5. Comparison of the results of the spectroscopic analysis of $\mathrm{Crh}$ pI-precipitates before and after temperature increase to those obtained for the Crh aggregates. In the upper panel, increased protein dynamics in the native pI-precipitate are indicated in gray according to Figure 2a. For Crh aggregates (lower panel, EM micrograph), red color-coding refers to chemical-shift changes and/or increased molecular dynamics. Secondarystructure elements, which can still be identified in the aggregate, are given in blue.

identified by a sequential walk from Ala65 to Ala79. These changes are in general small except for Gly67, which exhibits an upfield ${ }^{15} \mathrm{~N}$ shift of more than $10 \mathrm{ppm}$. In the domainswapped Crh fold, Gly67 NH forms a hydrogen bond to Glu70 $\mathrm{O} \epsilon$, stabilizing the turn between $\beta$-strand 4 and helix $\mathrm{C}$. In addition, substantial chemical-shift changes were seen for Asp69, which forms important hydrogen bonds to residues 30 and 31, as discussed above. These findings indicate that, while helix $\mathrm{C}$ remains largely intact upon aggregation, the $3 \mathrm{D}$ fold of the protein observed in its crystalline or pI-precipitated forms is no longer conserved in the Crh aggregates.

In addition, an overall reduction in helix content of $\mathrm{Crh}$ aggregates is reflected in the $\alpha$-helical Ala signal intensities that were reduced from $70 \%$ in Crh microcrystals to $40 \%$ in the aggregate. Concomitantly, we observed an increase in $\beta$-sheet and random-coil content of $20 \%$ and $10 \%$, respectively (see Supporting Information Figure 10). This increase in overall $\beta$-sheet content complicated an investigation analogous to the study regarding the three $\alpha$-helices for the $\beta$-strand regions of Crh aggregate. Nevertheless, we found that at least parts of $\beta$-strands $\beta 3$ and $\beta 4$ of $\mathrm{Crh}$ were preserved. Both segments, along with helix $\mathrm{C}$, are indicated in Figure 5 on the Crh X-ray structure in blue (lower panel). Interestingly, these segments are all located far from the hinge region. In addition, Figure 5 shows in red protein residues that exhibit chemical-shift alterations, meaning that the X-ray structure shown in the figure is no longer conserved in these regions upon aggregation. Remarkably, residues with isolated chemical shifts and located in loops or turns, such as Thr30, Ser31, Asp38, Ala54, Thr57/ 59, and Asp69, were clearly no longer observed at their initial chemical shift, indicating that the three-dimensional organization is profoundly altered, even of the conserved secondary structure elements. Finally, the outlier chemical shift for $\mathrm{Thr} 12 \mathrm{C} \alpha / \mathrm{C} \beta$, which is indicative for dimer formation, was not detected in the spectra of the aggregated form. This supports our conclusion that the conformation of the hinge region is substantially altered in the aggregated form and that protein regions essential for stabilization of the 3D fold of the Crh domain-swapped dimer have undergone major conformational changes.

\section{Discussion}

We have shown that ssNMR allows one to study the structure and identify the dynamics in a site-resolved manner in insoluble folding states. Hence, ssNMR not only allows to follow conformational transitions at specific molecular sites ${ }^{37}$ but, as demonstrated here, also enables the recording of time-dependent conformational changes in real time and at the level of individual residues in fully labeled proteins. Our data reveal that $\mathrm{Crh}$ pIprecipitates exhibit a higher degree of molecular mobility as compared to the crystalline state, with a core that is rigidly intact. Destabilization of this conformation by a moderate temperature increase was directly followed by $2 \mathrm{D}$ ssNMR. Accordingly, a partially unfolded intermediate state was created, which led to $\beta$-sheet rich protein aggregates containing a remarkable fraction of $\alpha$-helical segments.

Our observations confirm the previously postulated ${ }^{38}$ presence of intermediate states for domain-swapped proteins. Crh aggregates do not contain native domain-swapped proteins, which speaks against a run-away domain $\operatorname{swap}^{39,40}$ as a mechanism for oligomer formation in this protein. The conversion of a destabilized native protein state to a $\beta$-sheet rich aggregate was previously seen for a fibril-forming protein, ${ }^{41}$ where the aggregation process occurred without the need for dissolution and renucleation of the aggregates. This is compatible with our observation of "solid" intermediate states during the conformational transition in Crh, where aggregates are formed by a reorganization of the initial pI-precipitates, possibly assisted by the higher degree of flexibility observed in the precipitate. Dynamic behavior was also observed in a native-like fibril precursor of a naturally amyloidogenic protein, ${ }^{42}$ and the increased flexibility was linked to the interconversion of the native state and a folding intermediate.

Recently, ssNMR methods have shown their immense potential to study 3D molecular structure and dynamics (see, e.g., refs 43-45) using a single isotope-labeled sample. Application of these methods will offer additional means to

(37) Kamihira, M.; Naito, A.; Tuzi, S.; Nosaka, A. Y.; Saito, H. Protein Sci. 2000, 9, 867-877.

(38) Rousseau, F.; Schymkowitz, J. W.; Wilkinson, H. R.; Itzhaki, L. S. J. Biol. Chem. 2004, 279, 8368-8377.

(39) Nelson, R.; Eisenberg, D. Curr. Opin. Struct. Biol. 2006, 16, 1-6.

(40) Guo, Z.; Eisenberg, D. Proc. Natl. Acad. Sci. U.S.A. 2006, 103, 80428047.

(41) Plakoutsi, G.; Bemporad, F.; Calamai, M.; Taddei, N.; Dobson, C. M. Chiti, F. J. Mol. Biol. 2005, 351, 910-922.

(42) Jahn, T. R.; Parker, M. J.; Homans, S. W.; Radford, S. E. Nat. Struct. Mol. Biol. 2006, 13, 195-201.

(43) Luca, S.; Heise, H.; Baldus, M. Acc. Chem. Res. 2003, 36, 858-865.

(44) Giraud, N.; Böckmann, A.; Lesage, A.; Penin, F.; Blackledge, M.; Emsley, L. J. Am. Chem. Soc. 2004, 126, 11422-11423.

(45) McDermott, A. E. Curr. Opin. Struct. Biol. 2004, 14, 554-561. 
characterize structure and dynamics of protein refolding events that take place on a slow time scale and in dense molecular systems, as observed in vitro and in vivo for many neurodegenerative diseases.

Acknowledgment. We thank Dr. Henrike Heise for helpful discussions. This work was funded by the CNRS (PICS $n^{\circ}$ 2424), the French research ministry (ACI Biol. Cell. Mol. et Struct. 2003; ANR JCJC 2005), and the Max Planck Gesellschaft.
Supporting Information Available: NCA spectra, PDSD spectra of the carbonyl region of the precipitated form, and carbon chemical-shift differences and intensity ratios between the microcrystalline and precipitated forms; NCACX and NCOCX spectra of the aggregated form, and an analysis of the relative peak volumes for alanine residues in the microcrystalline and aggregated states. This material is available free of charge via the Internet at http://pubs.acs.org.

JA066469X 